\title{
An Integrated Model of Atom Search Optimization-Based Resonance Sparse Signal Decomposition and Cross-Validation SVM for Gearbox Fault Diagnosis
}

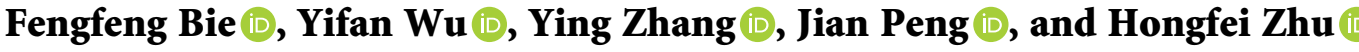 \\ School of Mechanical Engineering and Rail Transit, Changzhou University, Changzhou 213016, Jiangsu, China \\ Correspondence should be addressed to Fengfeng Bie; bieff@cczu.edu.cn
}

Received 15 October 2021; Revised 13 January 2022; Accepted 27 January 2022; Published 27 February 2022

Academic Editor: Baoping Cai

Copyright (c) 2022 Fengfeng Bie et al. This is an open access article distributed under the Creative Commons Attribution License, which permits unrestricted use, distribution, and reproduction in any medium, provided the original work is properly cited.

\begin{abstract}
In the aspect of gearbox fault diagnosis, the periodic pulse signal containing fault characteristics is often overwhelmed with other strong interference components, which brings a great challenge for gearbox fault detection and status identification. To address these issues, this paper develops a novel resonance-based sparse signal decomposition (RSSD) fault diagnosis method combined with support vector machine (SVM). Based on the key of the decomposition parameters in the resonant sparse decomposition method, the influence of atom search optimization (ASO) on the quality factor in the resonance-based sparse signal decomposition method is primarily studied. The vibration signal of the gearbox was decomposed by resonance sparse decomposition with the optimal quality factor, from which the high and low-resonance components were obtained. Then, the power spectrum entropy, singular spectrum entropy, and time-domain energy entropy of the low-resonance signal were calculated. Finally, the pattern recognition of gearbox fault was completed with a designed cross-validation SVM pattern recognition model. The numerical simulation and gearbox fault experiments demonstrate that the presented method can achieve great recognition accuracies and effect capacities against interference components involved in the gearbox vibration signal.
\end{abstract}

\section{Introduction}

As the core component of mechanical power and motion transmission, gearbox drives are widely used in metallurgical, petrochemical, mining, transportation, and other mechanical systems. The status monitoring and fault diagnosis of gearbox are critically important to ensure the reliability and efficiency of the mechanical system. In recent years, more and more attention has been paid to the research of gear state monitoring and fault diagnosis technology because of its relatively complex, nonlinear, and nonstationary dynamic behavior. Since the failure mode of the gearbox could be mostly figured out as periodic pulse characteristic, while the measured fault signals are bound to be overwhelmed with harmonic signals and background noise signals generated in the gearbox system, the core issue of the diagnosis process is highlighted as periodic pulse feature extraction and evaluation [1].
As mentioned in most related vibration-based studies, the fault diagnosis was processed in three main forms [2], i.e., the vibration separation, amplitude-frequency demodulation, and the advanced signal processing-based algorithms. Vibration separation-based algorithms are proposed to deal with the complex structure of the planetary gearbox aiming at eliminating the unrelated vibration from original signal through the time synchronized average (TSA) algorithm and its improved versions [3]. Another way for gearbox fault diagnosis was developed as identifying the amplitude and quantity increment of fault feature sideband around the meshing frequency and the corresponding harmonic in the spectrum. As the research focus, typical signal processing methods are developed in related fields [4], such as, empirical mode decomposition (EMD), ensemble empirical mode decomposition (EEMD), local mean decomposition (LMD), variational mode decomposition (VMD), and intrinsic time-scale decomposition (ITD) [5]. 
In addition, scholars have also made great progress on methods based on advanced signal processing, such as spectral kurtosis (SK)-based algorithm [6], continuous wavelet transformation (CWT) based on wavelet [7], algorithm adaptive stochastic resonance (ASR) based on stochastic resonance, and so on [8]. Besides, sparse representation-based algorithms are often used, as well as other processing methods, such as dynamic time warping (DTW), adaptive integrated empirical mode decomposition, and a series of algorithms [9]. In most of these algorithms, the waveform characteristics of the components are taken as decomposition gist, which is hard to work when the signal involves strong nonlinear nonstationary characteristics with background noise. To address this issue, resonance-based sparse signal decomposition is proposed [10], in which the vibration signal is decomposed into the high-resonance component through the tunable $Q$ wavelet transform, and the signal components with overlapping frequencies can be separated. This method breaks through the limitation of traditional decomposition method based on waveform and each component characteristic. When a defect emerges in the inner gear of the gearbox, periodic pulses are represented by corresponding fault vibration signals. Due to the coupling between the working environment and other mechanical components, the interference of noise and harmonic is involved in the collected gearbox vibration signal, which is shown as continuous oscillation, while the influence of the fault has lower oscillatory behavior unlike the harmonics and noise. RSSD has a more significant effect on feature extraction of nonlinear nonstationary signals of gearbox. A method based on resonance-based sparse signal decomposition integrated with empirical mode decomposition and demodulation was introduced in the literature [11] that can separate the impulse component from the signal, according to the different Q-factors of impulse component and harmonic component. According to the rotating speed signal, the time-domain impact signal of the gearbox was resampled at constant angle increments. Sun et al. [12] firstly applied the method of RSSD to the gearbox feature extraction process. With the frequency spectrum of the resampled collision signal being analyzed, the step domain analysis is completed, and the final diagnosis result is obtained accordingly. The proposed approach is of good anti-noise ability and is suitable for analyzing the actual vibration signal of a gearbox with rotating speed fluctuation. Tang et al. [13] proposed a fault diagnosis approach for planetary gearboxes using a multi-resonance component fusion-based convolutional neural network; RSSD is used to decompose the vibration signal, and the low-resonance components that may contain the impulse components of bearing faults can be retained. However, the final decomposition results might be greatly influenced by the choice of high-quality and lowquality factors for resonance-based sparse signal decomposition if the high-quality factor and low-quality factor are artificially determined, which is often inaccurate and even brings unreasonable final decomposition result. Thus, the ASO algorithm is adopted to determine the optimal quality factor in the study. The SVM model has been widely applied in mechanical fault pattern recognition, while for the process of gearbox fault recognition, the accuracy of SVM is regularly affected by $C$ (penalty factor) and $G$ (variance in RBF kernel function). Hence, the cross-validation method is employed to determine the final values of $C$ and $G$ to improve the accuracy of the final pattern recognition. Extensive shallow learning models, such as support vector machine (SVM), Naive Bayes classifier, and geometric model, were designed with the extracted inherent fault features from vibration signals [14]. SVM is widely used in the field of fault diagnosis.

Based on the above analysis, this paper proposes a new method for gearbox fault identification with improved RSSD and CV-SVM model. The main research contents include the following. The study of ASO made by the RSSD of low kurtosis of resonance components reached a maximum, and high-resonance decomposition is processed for the optimal parameters of $Q_{1}$ and $Q_{2}$ of low-resonance decomposition parameters. Then, power spectrum entropy, singular spectrum entropy, and time-domain energy entropy are analyzed in the feature extraction of low-resonance components for pattern recognition. The $C / G$ parameters of SVM are optimized with cross-validation, and the accuracy of pattern recognition is promoted with this method. The simulated fault signal is obtained through the digital model for the gear fault. Through model simulation, the simulated fault signal is obtained, and the feasibility of the method is verified. The rest of this paper is organized as follows. Section 2 states some theoretical background and presents the fault diagnosis process. Section 3 presents the details of gearbox fault simulation. In Section 4, the feasibility and superiority of this method are verified on three different types of gearbox fault data and compared with the nonoptimized diagnosis technology. Finally, the conclusion and future jobs are summarized in Section 5.

\section{Algorithm Principle}

2.1. RSSD Optimization Based on ASO. In general, vibration signals can be decomposed into three signals by RSSD, i.e., the high-resonance signal series with continuous oscillating behavior, the low-resonance components of transient impact signals without specific shape and duration, and the remaining residual component of the original signal. This is achieved by the tunable Q-factor wavelet transform (TQWT). The greater quality factor $Q$ value is obtained for the better aggregation performance on frequency domain of the high-resonance signal. Low-resonance signal has good time-domain aggregation, and its quality factor $Q$ value obtained is smaller [15]. The multi-channel bandpass decomposition filter banks are employed in the process of the tunable quality factor wavelet transform on the acquired gearbox vibration signals, and the basis function libraries of high Q-transform and low Q-transform are obtained. The principle of the method is shown in Figure 1, where $H_{0}(\omega)$ represents the low-resonance filter, $H_{1}(\omega)$ represents the high pass filter, $V_{0}(n)$ and $V_{1}(n)$ are the sub-band signals after filtering, and $\alpha$ and $\beta$ represent the low-pass and highpass scale factors, respectively. The quality factor $Q$, redundancy factor $R$, and the maximum number of 
decomposition layers $L_{\max }$ can be set referring to equations (1)-(3), [16].

$$
\begin{aligned}
Q & =\frac{2-\beta}{\beta}, \\
r & =\frac{\beta}{1-\alpha}, \\
L_{\max } & \leq \frac{\log (\beta N / 8)}{\log (1 / \alpha)} .
\end{aligned}
$$

Therefore, the acquired vibration signal $x$ can be expressed as the composition of the high-resonance component $x_{1}$, the low-resonance component $x_{2}$, and the participating component $n$, which can be expressed as [17]

$$
x=x_{1}+x_{2}+n,
$$

where $x_{1}$ and $x_{2}$ can be represented by overcomplete basis function libraries $S_{1}$ and $S_{2}$, which can be constructed by high-quality factor $Q_{1}$ and low-quality factor $Q_{2}$, respectively, and the objective function can be established by constructing MCA equation [18]:

$$
J\left(W_{1}, W_{2}\right)=\left\|x-S_{1} W_{1}-S_{2} W_{2}\right\|_{2}^{2}+\lambda_{1}\left\|W_{1}\right\|_{1}+\lambda_{2}\left\|W_{2}\right\|_{1},
$$

where $W_{1}$ and $W_{2}$ are the transformation coefficients under the basis function libraries $S_{1}$ and $S_{2}$, respectively, and $\lambda_{1}$ and $\lambda_{2}$ are the regularization parameters.

Equation (5) can be reorganized as

$$
J\left(W_{1}, W_{2}\right)=\left\|x-S_{1} W_{1}-S_{2} W_{2}\right\|_{2}^{2}+\sum_{j=1}^{J_{1}+1} \lambda_{1, j}\left\|W_{1, j}\right\|_{1}+\sum_{j=1}^{J_{1}+1} \lambda_{2, j}\left\|W_{2, j}\right\|_{1} .
$$

To achieve the minimum function value, the separation augmented Lagrange contraction algorithm (SALSA) is adopted for iterative calculation.

In resonance-based sparse signal decomposition, the choice of quality factors $Q_{1}$ and $Q_{2}$ plays a decisive role in the final decomposition result. The prior knowledge for the selection of quality factor parameters is often needed by the traditional resonance-based sparse signal decomposition method, which greatly increases the cost of operation. To solve the above problems, this paper proposes a quality factor optimization scheme based on ASO. The kurtosis of low-resonance component decomposed by RSSD can be taken as the objective function by this method. The optimal quality factor generates as the kurtosis value reaches the maximum level, since the value of kurtosis could correspondingly reflect the normal distribution of vibration signal [19]. The larger the noise, the smaller the kurtosis value and the less obvious the response. Kurtosis can be used as an important index to measure the number of pulse components in the signal. In the literature [19], kurtosis is effectively applied as an important index to measure the fault of reciprocating compressor. The expression of kurtosis is as follows:

$$
K=\frac{E(x-\mu)^{4}}{\sigma^{4}},
$$

where $\mu$ represents the mean value of the vibration signal, $\sigma$ represents the standard deviation of the vibration signal, and $E$ is the mathematical expectation.

Atom search optimization is a novel intelligent algorithm based on molecular dynamics model proposed in 2019 [20]. In a molecular system, there are interaction forces (attraction and repulsion) between neighboring atoms, and the globally optimal atoms have geometric constraints on other atoms. Gravitation urges atoms to explore the whole search space extensively, and repulsion enables them to develop potential regions effectively. It has the characteristics of strong optimization ability and fast convergence.

The acceleration of the $i-$ th atom at time $t$ can be expressed as

$$
\begin{aligned}
& a_{i}^{d}(t)=\frac{F_{i}^{d}(t)}{m_{i}^{d}(t)}+\frac{G_{i}^{d}(t)}{m_{i}^{d}(t)}=-\delta\left(1-\frac{t-1}{T_{\max }}\right)^{3} e^{-\left(20 t / T_{\max }\right)}, \\
& \sum_{j \in \text { Kbest }} \frac{\operatorname{rand}_{j}\left[2 \times\left(h_{i j}(t)\right)^{13}-\left(h_{i j}(t)\right)^{7}\right]}{m_{i}(t)}, \\
& \frac{\left(x_{i}^{d}(t)-x_{j}^{d}(t)\right)}{\left\|x_{i}(t), x_{j}(t)\right\|}+\gamma e^{-\left(20 t / T_{\max }\right)} \frac{x_{\text {best }}^{d}(t)-x_{i}^{d}(t)}{m_{i}(t)},
\end{aligned}
$$

where $\delta$ represents depth weighting, $T_{\max }$ represents the maximum number of iterations, $h_{i j}(t)$ represents the distance between the two atoms, $\gamma$ is the multiplier weight, and $m_{i}(t)$ is the mass of the $i-$ th atom after the $t-$ th iteration. The velocity and position of the atomic movement can be changed by the acceleration of atoms:

$$
\begin{aligned}
v_{i}^{d}(t+1) & =\operatorname{rand}_{i}^{d} v_{i}^{d}(t)+a_{i}^{d}(t), \\
x_{i}^{d}(t+1) & =x_{i}^{d}(t)+v_{i}^{d}(t+1),
\end{aligned}
$$

where $x_{i}^{d}(t)$ is the position of the $i$ - th atom after $t$ - th iterations, $v_{i}^{d}(t)$ is the velocity of the $i-$ th atom after $t-$ th iterations, and $a_{i}^{d}(t)$ is the acceleration of the $i$ - th atom after $t$ - th iterations.

In order to improve the global search ability of ASO algorithm in the initial iteration, every atom should interact with as many other optimal atoms as possible. To improve its search ability in the later iteration, every atom should interact as few as possible. 


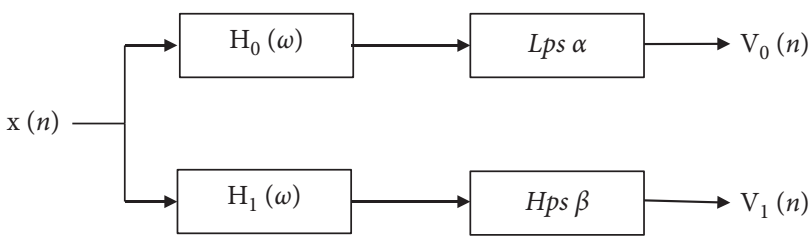

(a)

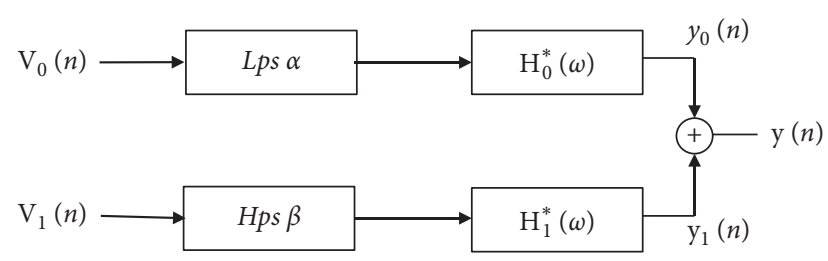

(b)

Figure 1: Bandpass filter banks. (a) Decomposition filter banks. (b) Reconstructed filter.

2.2. Cross-Validation SVM. SVM is usually used to classify problems with multiple categories [21]. The SVM method was first applied to the fault diagnosis field of gearbox, and its fault classification effect is relatively ideal. In reference [22], it is proved that the cross-validation method can effectively improve the classification accuracy of SVM, and this method is significantly better than the traditional SVM method. In order to obtain a better classification effect, CVSVM was used for pattern recognition of single fault of gearbox.

For a training sample $\left\{\left(x_{1}, y_{1}\right),\left(x_{2}, y_{2}\right), \ldots,\left(x_{k}, y_{k}\right)\right\}$ set with a sample number of $k$, where $x_{i} \in R^{d}$ represents the input index and $y_{i} \in\{-1,1\}$ represents the output index (where $i=1,2, \ldots, k$ ), if the optimal hyperplane is set to $w * x+b=0$, the weight vector $w$ and bias $b$ must satisfy the following constraints:

$$
y_{i}(w * x+b) \geq 1-\zeta_{i}
$$

where $\zeta_{i}$ is the relaxation variable and represents the degree of deviation between the SVM classification model and the ideal linear case. The hyperplane decision function of the linear classifier is

$$
M(x)=\operatorname{sgn}\left\{\sum_{i}^{n} c_{i}^{*} y_{i}(\chi \bullet \chi)+b^{*}\right\},
$$

where sgn represents the function, $c_{i}^{*}$ represents the optimal solution of $c_{i}, b^{*}$ is the threshold value of classification, $\chi$ is the samples to be classified, and $M(x)$ represents the classification function, where the category of the classification sample $\chi$ is judged according to the positive or negative value of the classification function.

Cross-validation is a process commonly applied in validating the performance of a given classifier, in which the data are firstly grouped, and then the validation set is composed of the training set and the corresponding training parameters [23]. The best parameter $C$ and parameter $G$ can be found with cross-validation for the selection of the best parameter of the training model. In the process of vibration and impact feature extraction of gearbox, in order to extract the most significant fault feature as much, the entropy feature should be extracted from multiple ways. Reference [24] elaborates the advantages of extracting entropy features from multiple aspects over extracting entropy features from a single point. Meanwhile, singular spectral entropy, timedomain energy entropy, and power spectral entropy could reflect signal features in time domain and frequency domain. Therefore, only three entropy features are adopted in this paper to construct feature vectors. The complexity and uncertainty of frequency domain signal can be reflected by power spectrum entropy. The more evenly the energy in the signal is distributed in the frequency domain, the more complex the signal is and the higher the degree of uncertainty is. As a kind of information entropy, singular spectrum entropy reflects the uncertainty degree of each mode of time-domain signal under singular spectrum division [25]. As the gearbox fails, a periodic excitation generates in the gearbox system that could be involved in the gearbox vibration. The periodic excitation in the time-domain signal and the complexity and uncertainty of the time-domain signal energy can be manifested with the time-domain energy entropy. All the symbols and variables are explained in the nomenclature.

\subsection{Gear Fault Diagnosis Process Based on RSSD Optimized by} ASO Algorithm Combined with CV-SVM. The specific process is as follows.

Step 1: The maximum decomposition level $L_{1}$ of highresonance component is set as 30 , and the maximum decomposition level $L_{2}$ of low-resonance component is 12 ; the redundancy $r_{1}$ of high-resonance component is 9 , and the redundancy $r_{2}$ of low-resonance component is 3 .

Step 2: Given the initial high-resonance quality factor $Q_{1}$ value 4 and low-resonance quality factor $Q_{2}$ value 2 , the values of the high Q-factor and low Q-factor are set to be $0.8-1.3$ and 3-9, respectively.

Step 3: Set the population number of the ASO to be 50, the maximum iteration times are 1000 times, the depth weight is 50 , and the multiplier weight is 0.2 .

Step 4: Set the target function as the kurtosis of low-resonance component. When the kurtosis reaches the maximum value of iteration, the process stops and the $Q_{1}$ and $Q_{2}$ are output. Otherwise, repeat Steps 2 and 3.

Step 5: To obtain the low-resonance component and highresonance component, the power spectrum entropy, singular spectrum entropy, and time-domain energy entropy of the low-resonance component are calculated. These three kinds of feature entropy are substituted into SVM of crossvalidation to recognize patterns.

The specific process is shown in Figure 2. 


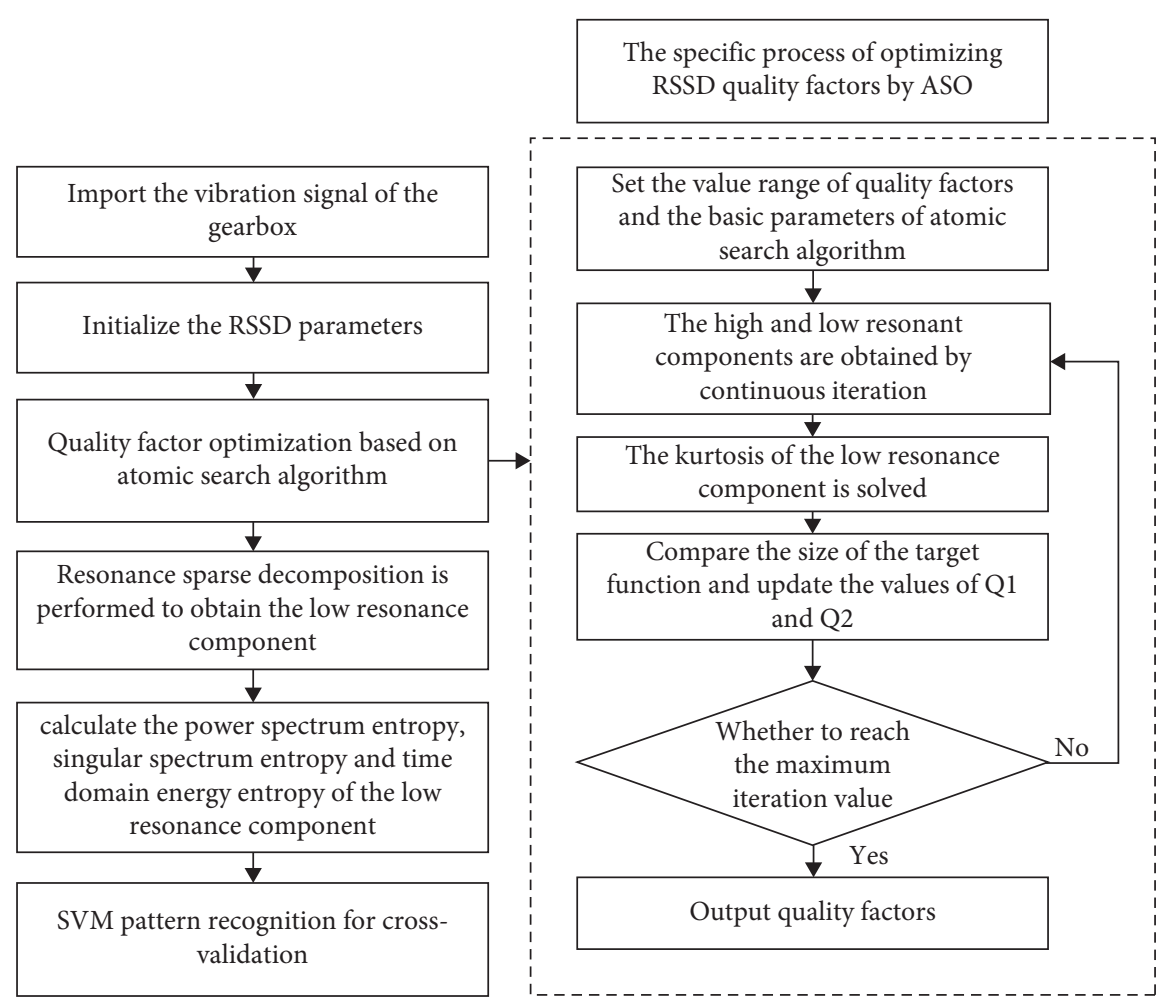

FIGURE 2: Flowchart of fault diagnosis and pattern recognition of gearbox with resonance-based sparse signal decomposition based on ASO.

TABLE 1: Parameters of spur bevel gears.

\begin{tabular}{lcc}
\hline Parameter & Driving gear & Driven gear \\
\hline Number of teeth & 17 & 27 \\
Diametral pitch & 10 & 10 \\
Reference circle & 1.7 & 2.7 \\
Pressure angle & 20 & 20 \\
Reference cone angle & 32.2 & 57.8 \\
Face width & 0.5 & 0.5 \\
Addendum & 0.13 & 0.07 \\
Dedendum & 0.09 & 0.15 \\
Tip angle & 37.44 & 61.07 \\
Root angle & 28.93 & 52.56 \\
\hline
\end{tabular}

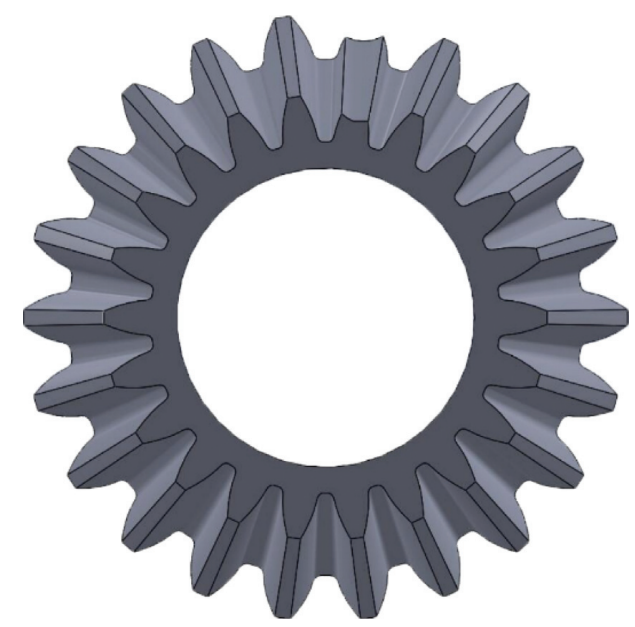

Figure 3: Tooth fracture of driving gear. 


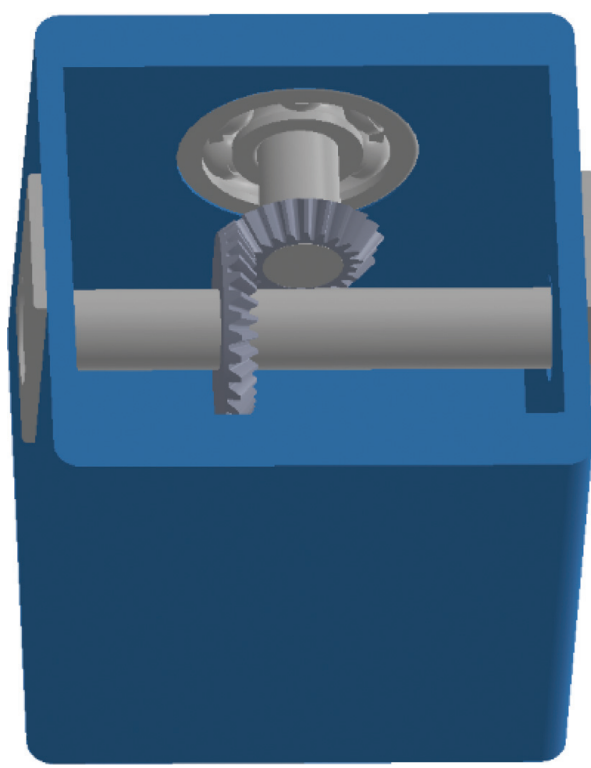

FIgURE 4: Structure of the gearbox.

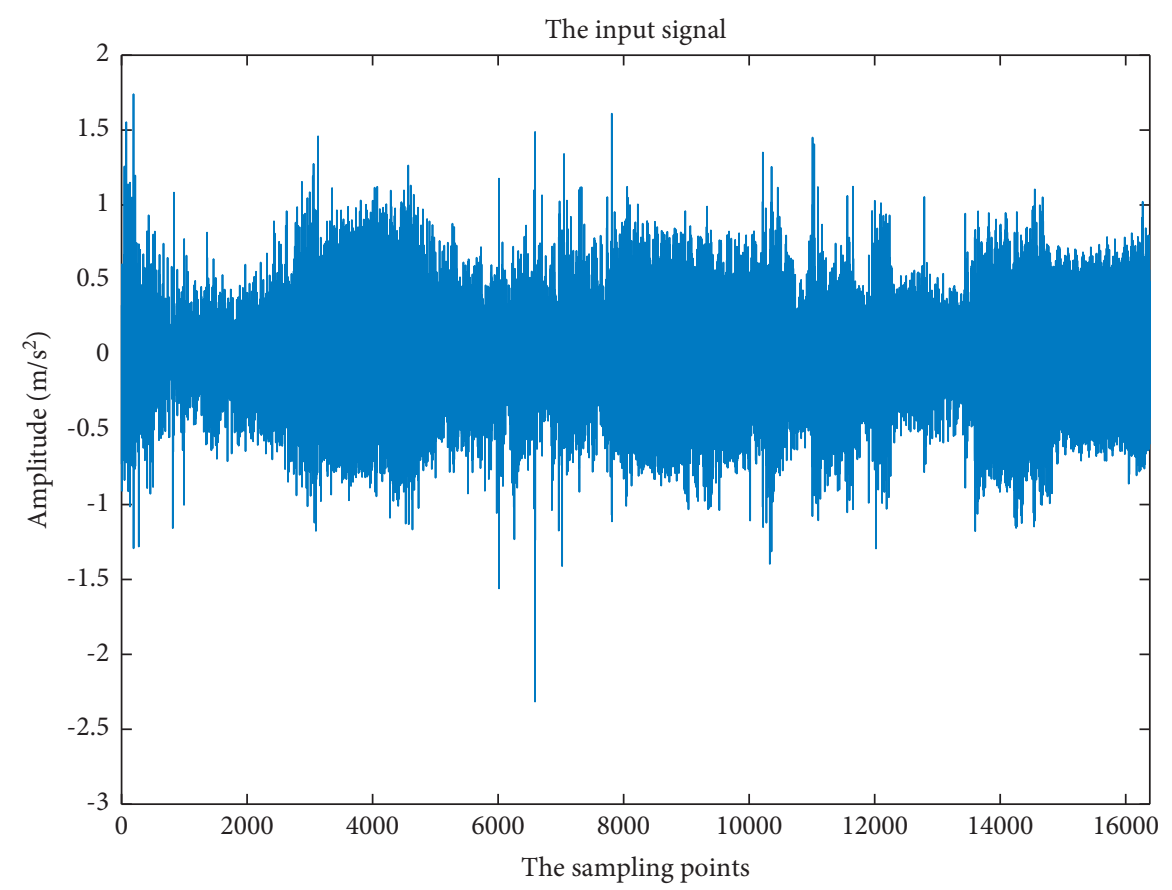

Figure 5: Time-domain simulation signal.

\section{Analogue Simulation}

In order to verify the rationality of the method proposed above, a single fault model of gearbox is established. The research object of this paper is a straight bevel gear, whose structural parameters are shown in Table 1: the face width coefficient $\Phi R=0.3$, the coefficient of addendum height $h a^{*}=1.0$, and the coefficient of clearance $c^{*}=0.2$. The failure of driving gear is shown in Figure 3. According to the parameter set, the system of 3D model of gear, gear shaft, bearing, and gearbox is established and assembled by SolidWorks. The structural parameters are the same as those of the experimental gearbox shown in Figure 4.

The model is imported into ADAMS for simulation. The sensor is set above the meshing position of the gear. The data of radial vibration of gearbox were measured. In order to make the simulation signal more realistic, the case of the gearbox box is set to be flexible before the simulation starts, the simulation time is $1.6 \mathrm{~s}$, and the step is set to 2000 . The time-domain diagram of the final simulation signal is shown in Figure 5. 

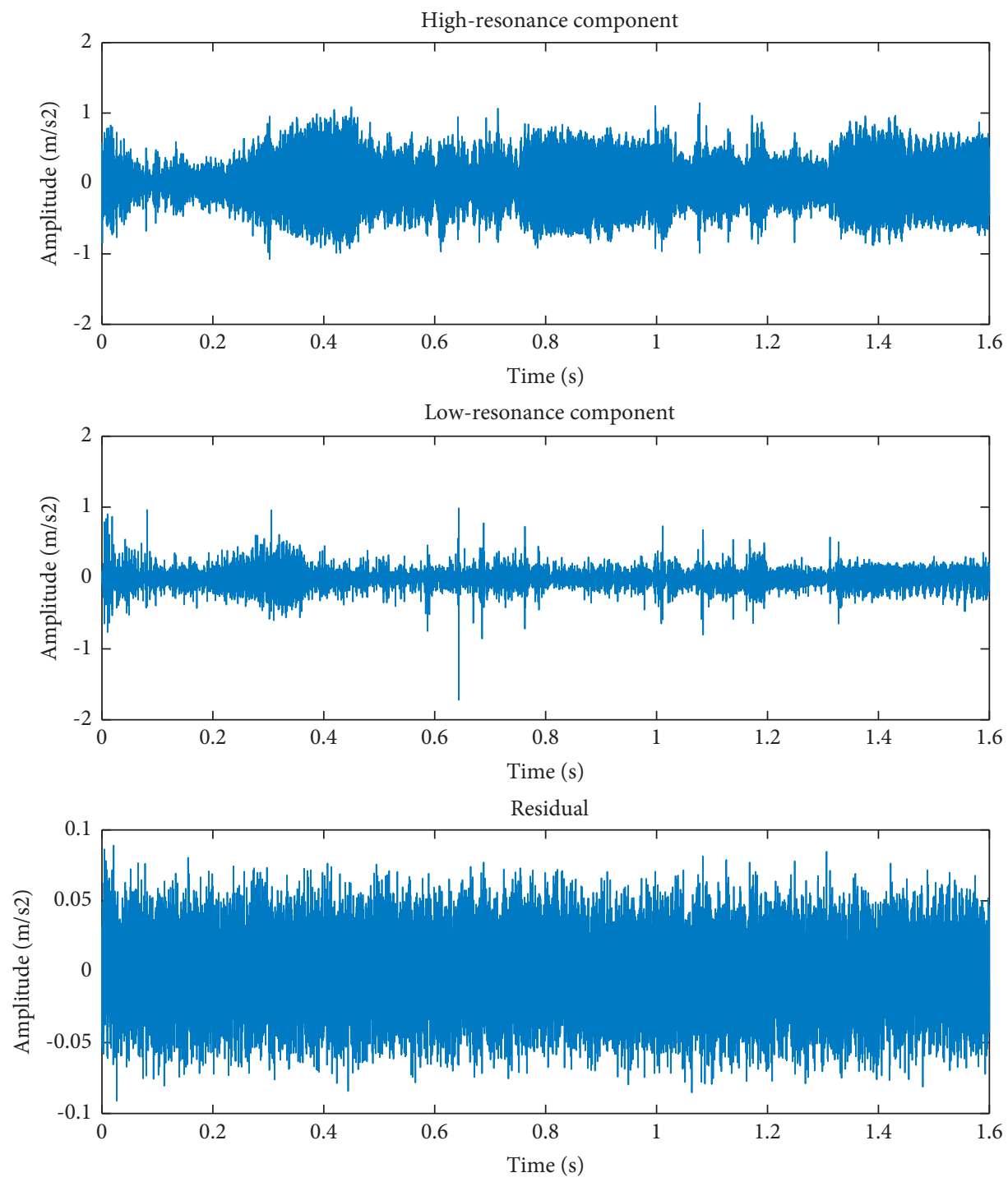

FIgURE 6: Resonance-based sparse signal decomposition with kurtosis as optimization objective.

The ASO is employed to find the optimal high Q-factor $Q_{1}$ and low $Q$-factor $Q_{2}$. After the iterative search of atom search algorithm, the high $Q$-factor $Q_{1}=7.1767$ and the low $\mathrm{Q}$-factor $Q_{2}=1.2184$ are finally determined. In the subsequent resonance-based sparse signal decomposition, $Q_{1}$ and $Q_{2}$ are applied to process the simulation signal. The highresonance component, low-resonance component, and residual component are obtained as shown in Figure 6 . Conventional parameters using the time domain of the vibration signal (kurtosis and skewness) were used for detecting and diagnosing the faults by applying them to filtered signals [26]. In order to compare the results of kurtosis and skewness as optimization objectives, RSSD with skewness as optimization objective is applied to decompose the simulation signal, and the decomposition result is shown in Figure 7.

Figure 8 illustrates that the obvious peaks could be found at the meshing frequency and higher harmonics. For example, the amplitude reaches $0.008288 \mathrm{~m} / \mathrm{s}^{2}$ at $f_{c}=1146 \mathrm{~Hz}$, which obviously indicates the local abnormality of the gearbox. The spectral lines of 1,2 , and 3 times the conversion frequency show that the modulation frequency of the impact pulse reaches the maximum value, which is consistent with the rotation frequency of the gear shaft; therefore, tooth fracture mode of the gearbox could be identified. Envelope spectrum of RSSD with skewness as optimization objective is shown in Figure 9. From the comparison of envelope spectra of these two typical indicators, it can be obviously observed that the RSSD with kurtosis performs better in the signal decomposition process.

\section{Experimental Validation}

As shown in Figures 10(a) and 10(b), the experimental system is established for the validation of the proposed method based on SQI-MFS mechanical fault simulation platform. The sensor is set above the meshing position of the gear, the motor frequency is set to $15 \mathrm{~Hz}$, and the sampling frequency is set to $5120 \mathrm{~Hz}$. The radial vibration signal of 

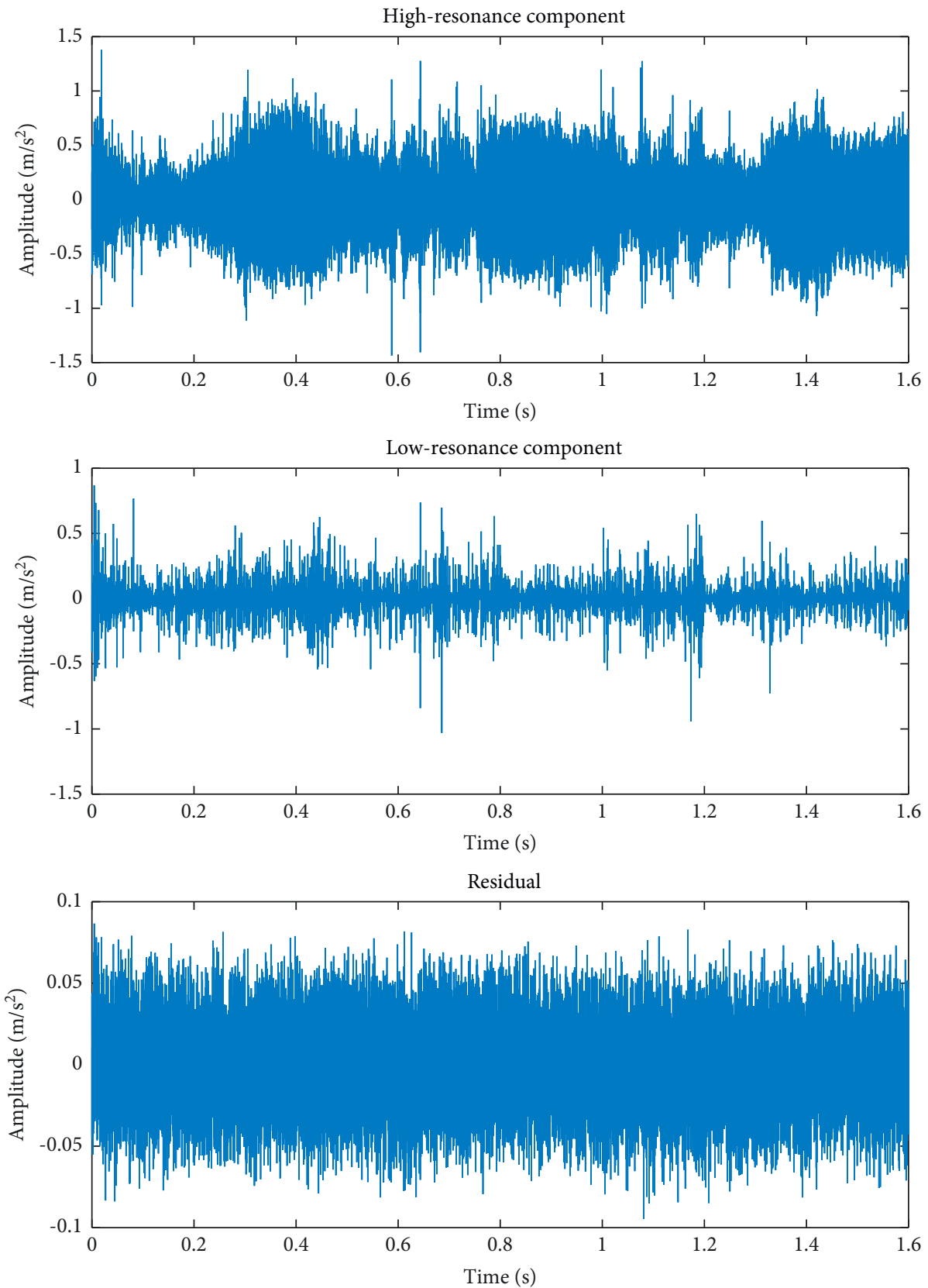

FIGURE 7: Resonance-based sparse signal decomposition with skewness as optimization objective.

gearbox is measured. Three typical failure modes of the gearbox are simulated, i.e., as abrasive, notched gear, and tooth fracture. The fault setting of the driving gear is shown in Figure 11.

The collected vibration signals of normal gearbox, abrasion, notched gear, and tooth fracture are shown in Figure 12. In the normal mode, the vibration amplitude is small and stable without obvious pulse feature, while in the other three fault cases, the amplitude increases significantly, and the notched gear's signal is particularly obvious and its impact component in the signal is also distinctive.

The resonance-based sparse signal decomposition parameters of the three kinds of failure signals were optimized by ASO. In the case of abrasion, the original high Q-factor $Q_{1}$ was set to 4 , and the low $Q$-factor $Q_{2}$ was set to 2 initially. With the ASO optimized, when the high Q-factor $Q_{1}=7.2767$ and the low $Q$-factor $Q_{2}=1.2184$, the kurtosis of the low-resonance component can reach the maximum. Similarly, $Q_{1}=6.8516$ and $Q_{2}=1.0297$ for notched gear, and $Q_{1}=6.9860$ and $Q_{2}=1.0004$ for tooth failure. The optimal quality factor is used for resonance sparse decomposition, and the results are shown in Figure 13.

The envelope spectrum of the low-resonance component obtained by the resonance-based sparse signal decomposition is shown in Figure 14. The existence of faults can be observed. However, the specific fault type cannot be well distinguished. In view of this, the entropy 


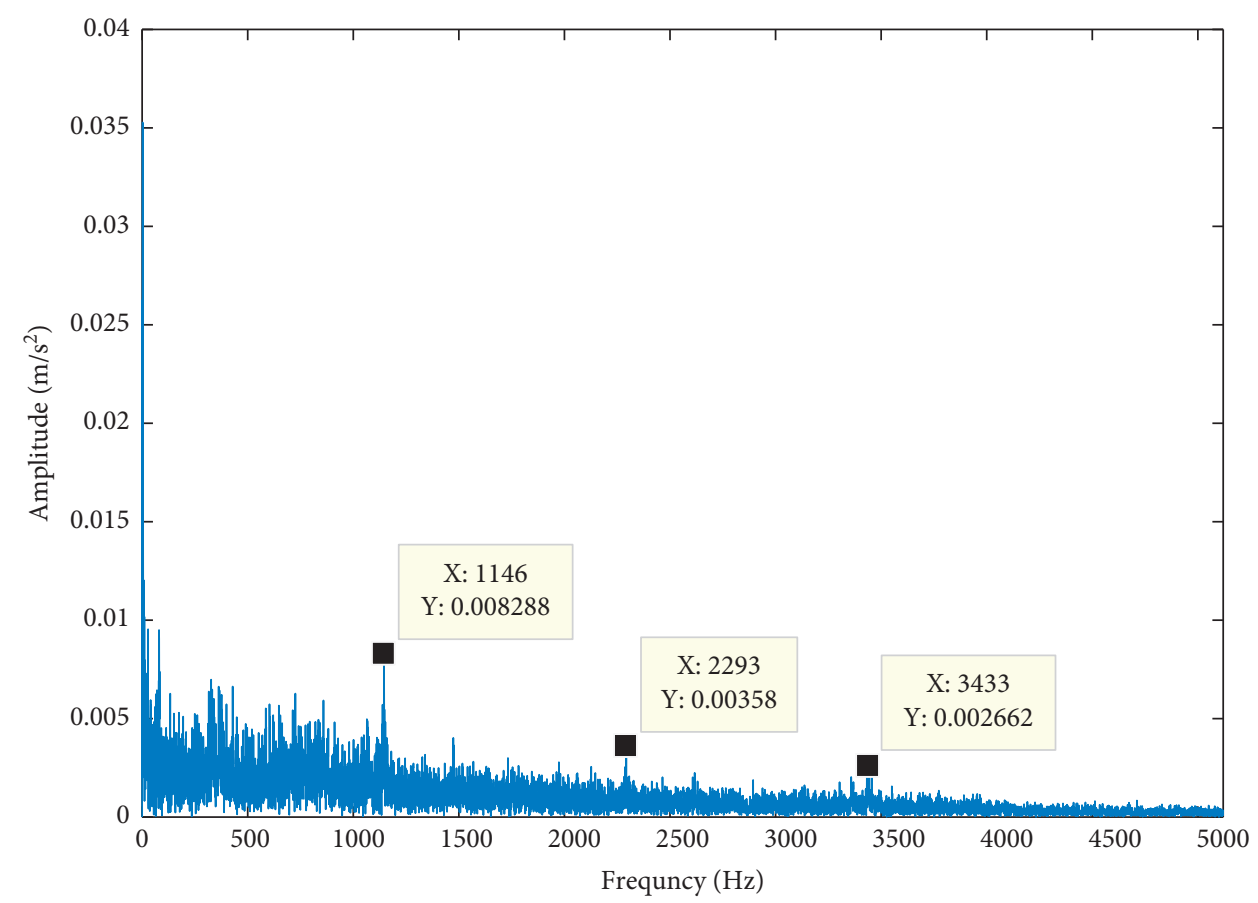

FIGURE 8: Envelope spectrum of the low-resonance component with kurtosis as optimization objective.

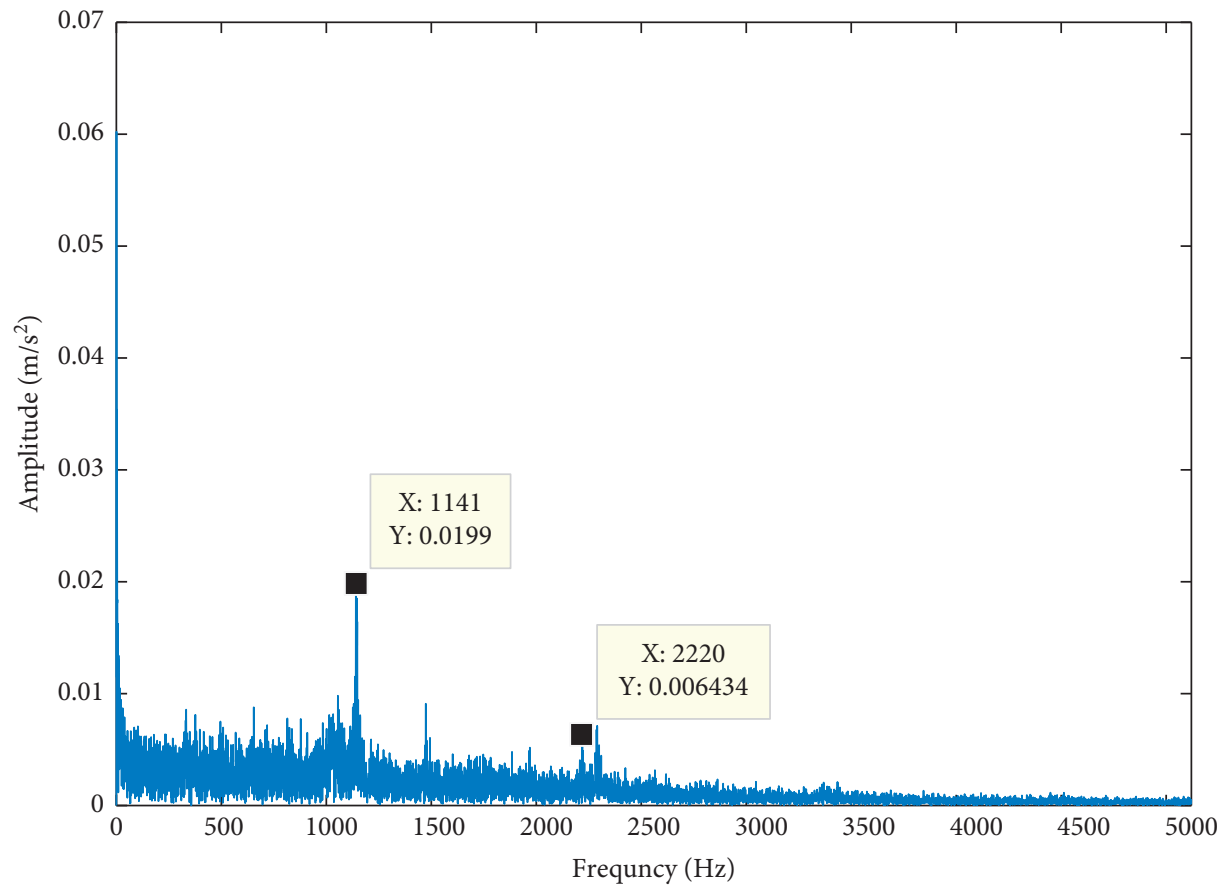

FIGURE 9: Envelope spectrum of the low-resonance component with skewness as optimization objective.

feature of each group of fault signals is extracted for CVSVM.

30 groups of vibration signals of normal, abrasion, notched gear, and tooth fracture are extracted, respectively, and the resonance-based sparse signal decomposition based on the optimization of ASO is processed. As the resonancebased sparse signal decomposition of various fault signals is optimized, the low-resonance components are obtained, and their power spectrum entropy, singular spectrum entropy, and time-domain energy entropy are extracted as the eigenvalues, respectively. 36 groups of data were randomly selected as the training set from 120 groups, and the remaining 84 were selected as the testing set. The normal state was marked as +1 , the wear state was marked as +2 , the missing tooth state was marked as +3 , and the broken tooth state was marked as +4 . The results of CV-SVM fault 


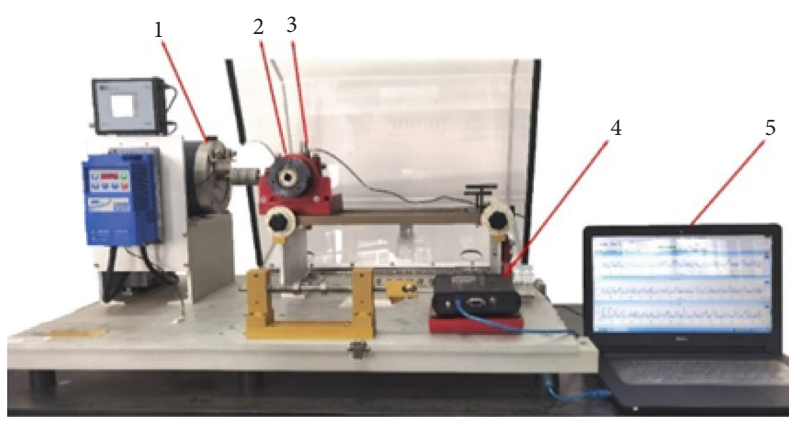

(a)

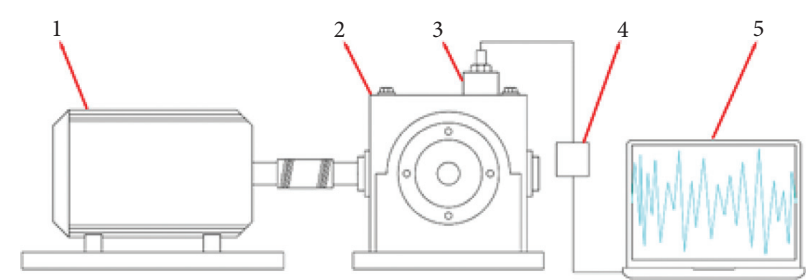

(b)

Figure 10: 1, motor drive; 2, gearbox; 3, acceleration sensor; 4, data acquisition instrument; 5, laptop and signal analysis software. (a) Test rig. (b) Data acquisition system sketch.

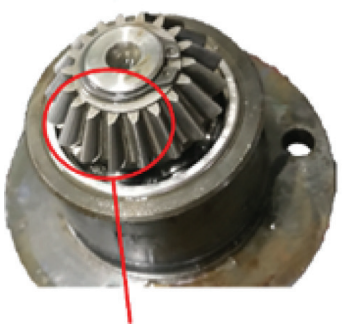

Abrasion

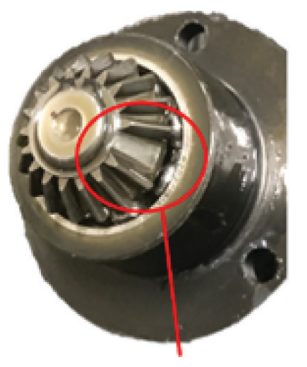

Notched gear

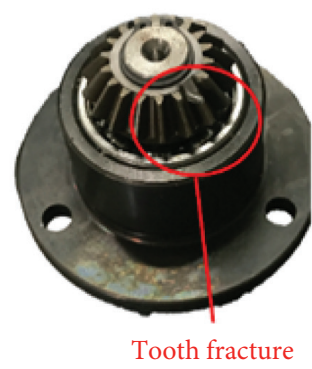

Tooth fracture

Figure 11: Failure mode setting.

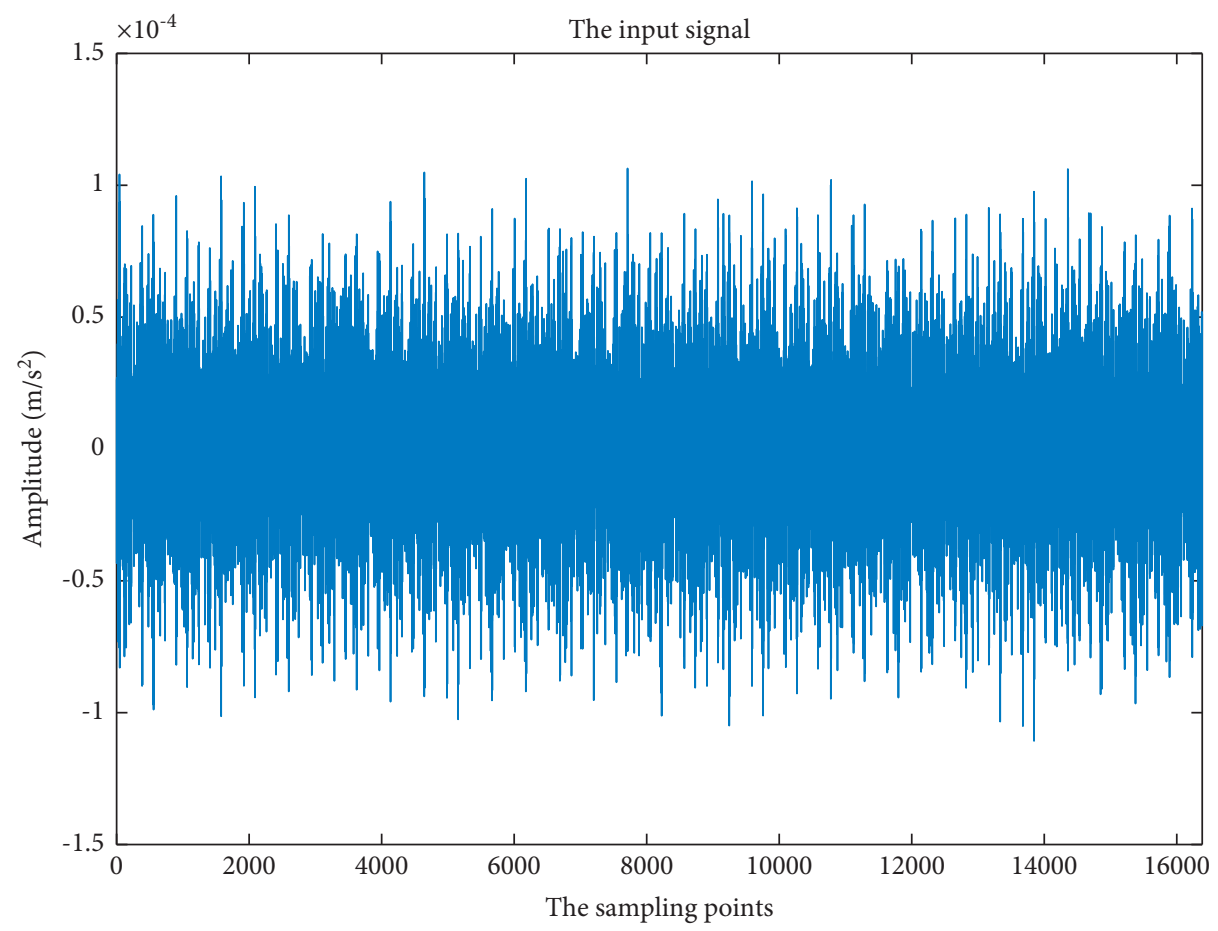

(a)

Figure 12: Continued. 


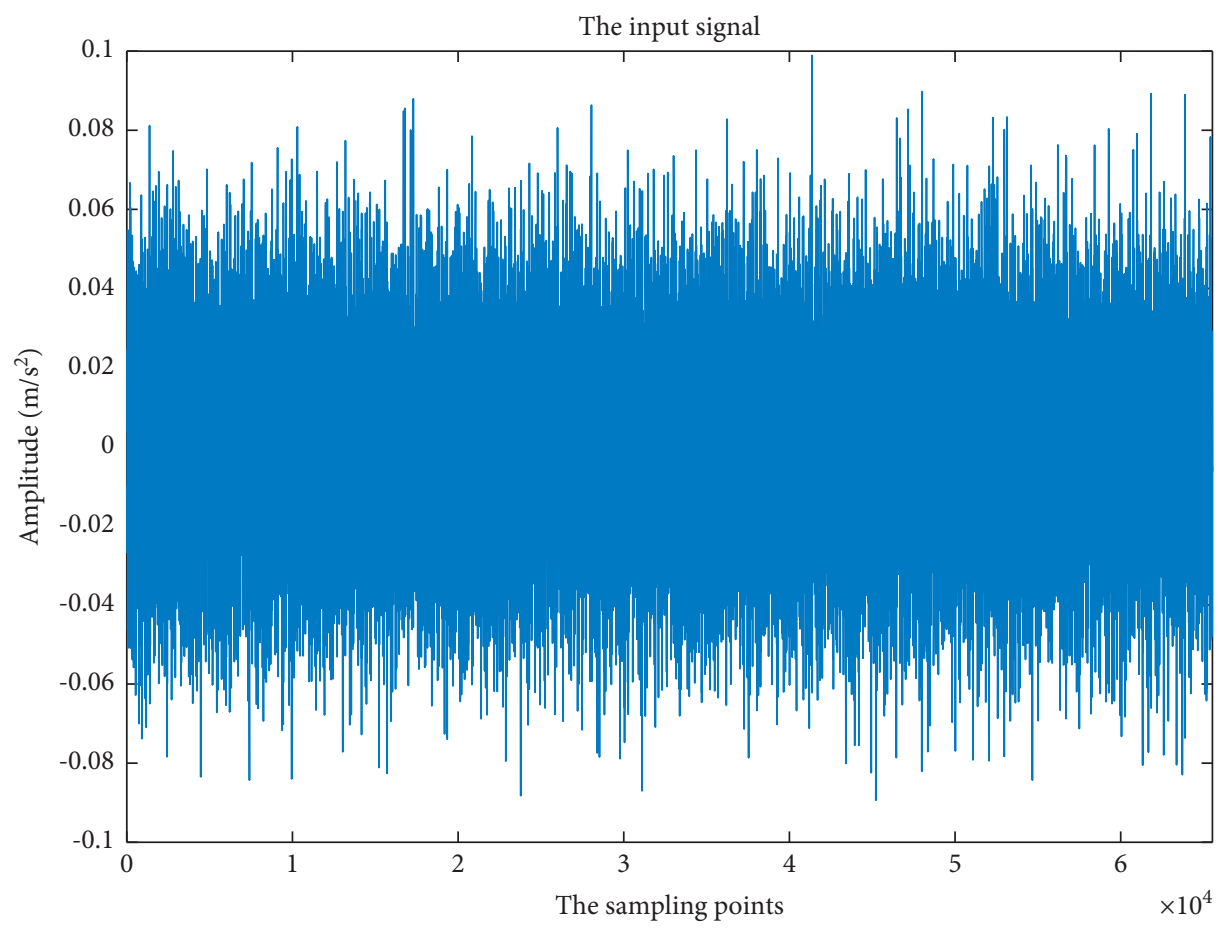

(b)

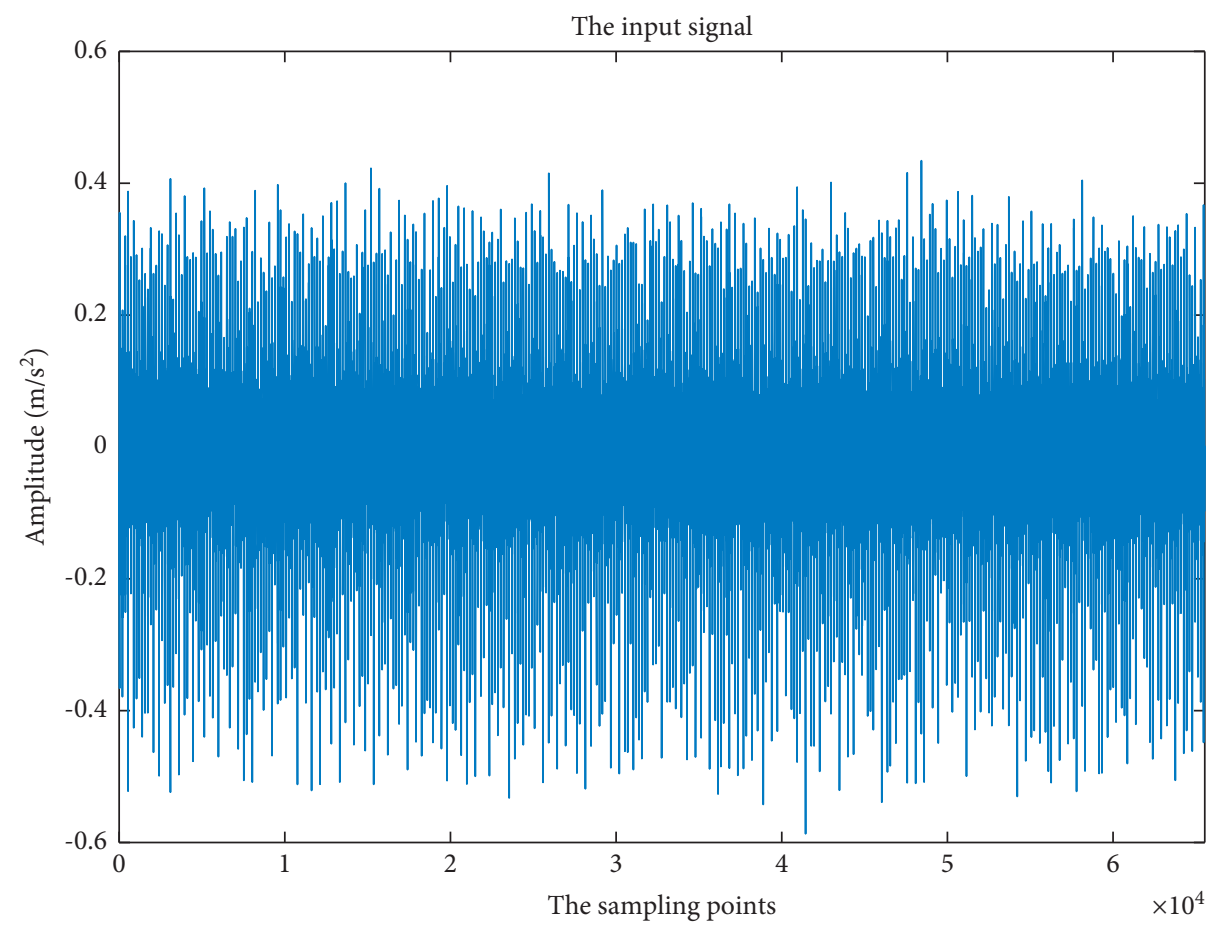

(c)

Figure 12: Continued. 


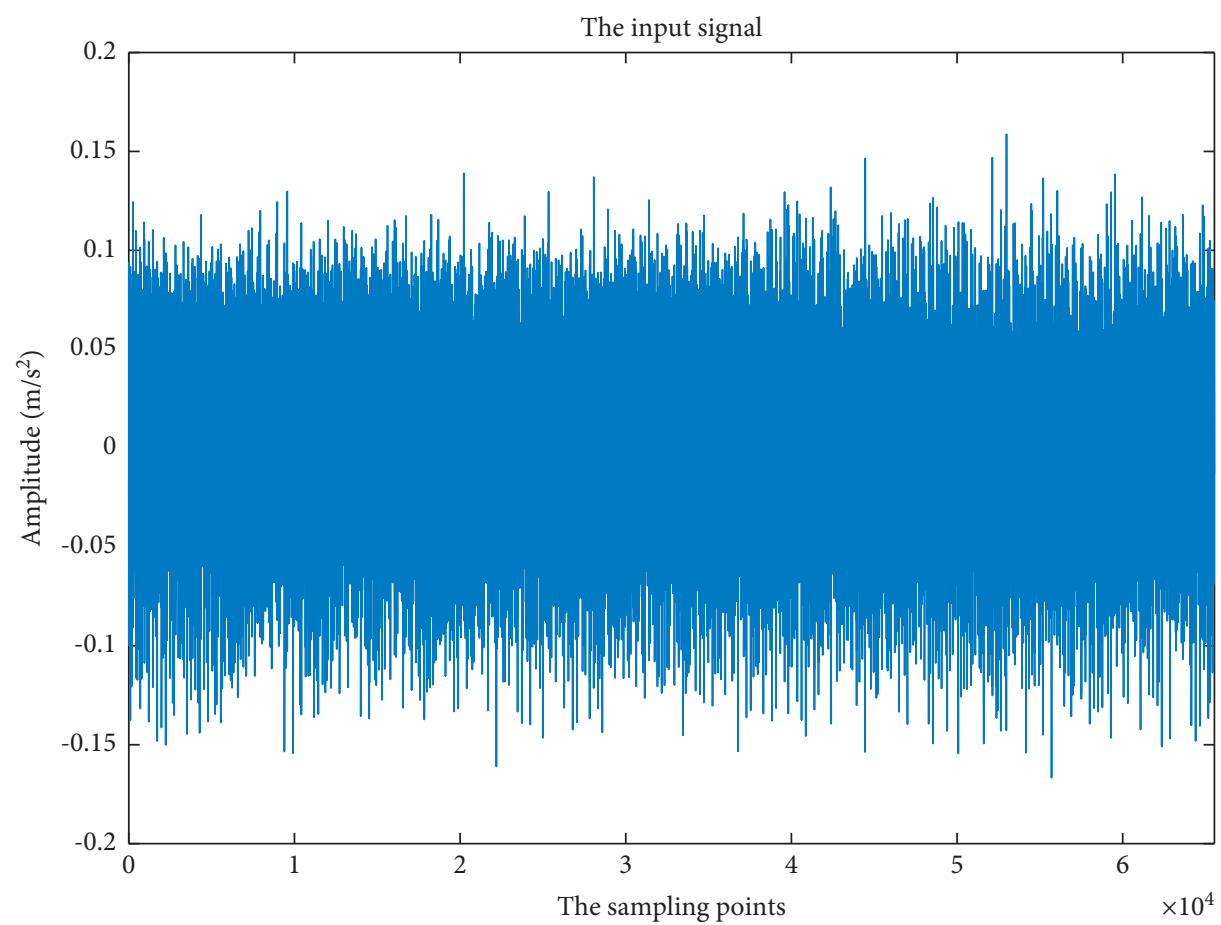

(d)

Figure 12: The collected vibration signals. (a) Time-domain signal of normal gear. (b) Time-domain signal of abrasion. (c) Time-domain signal of notched gear. (d) Time-domain signal of tooth fracture.
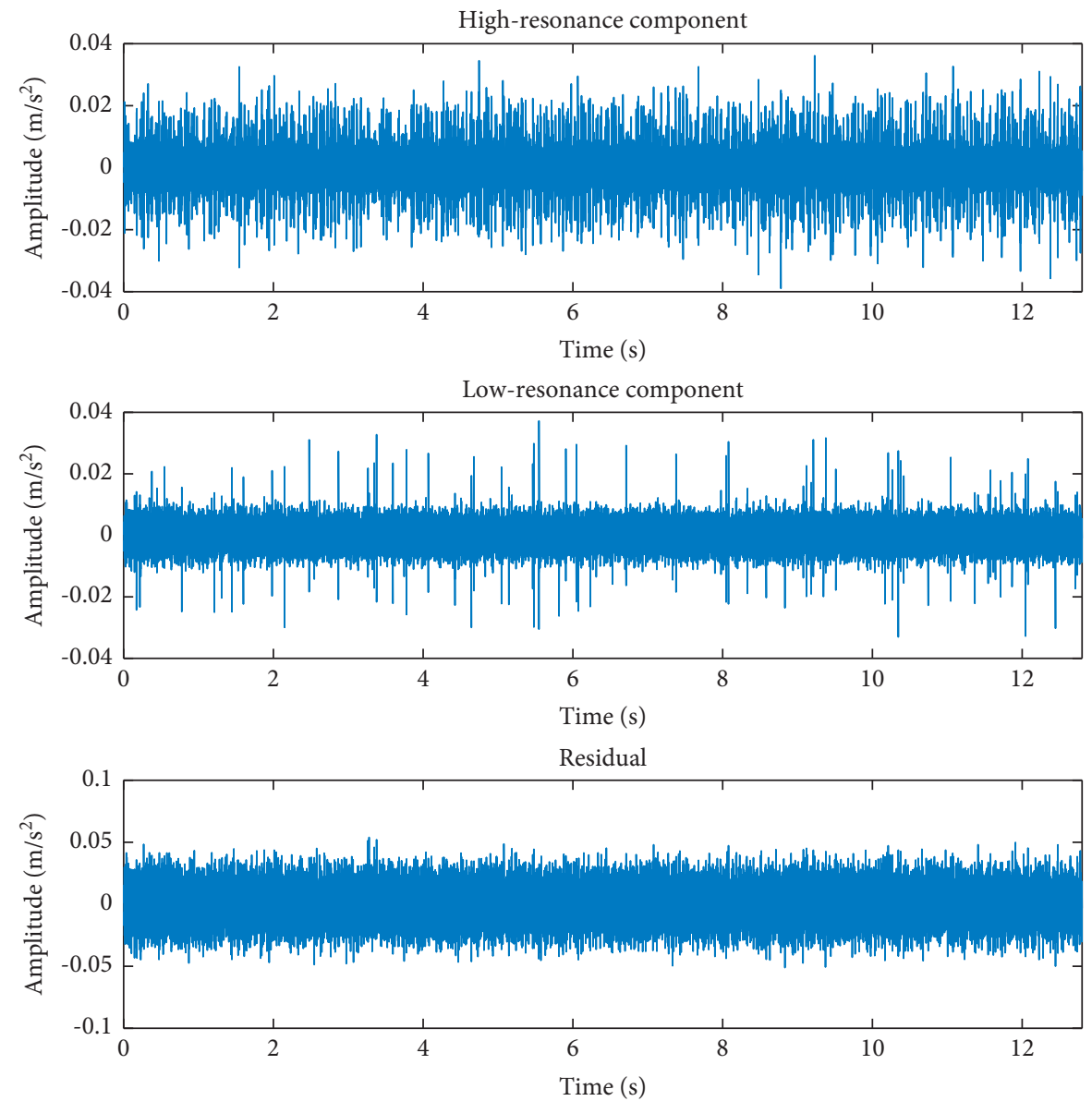

(a)

Figure 13: Continued. 

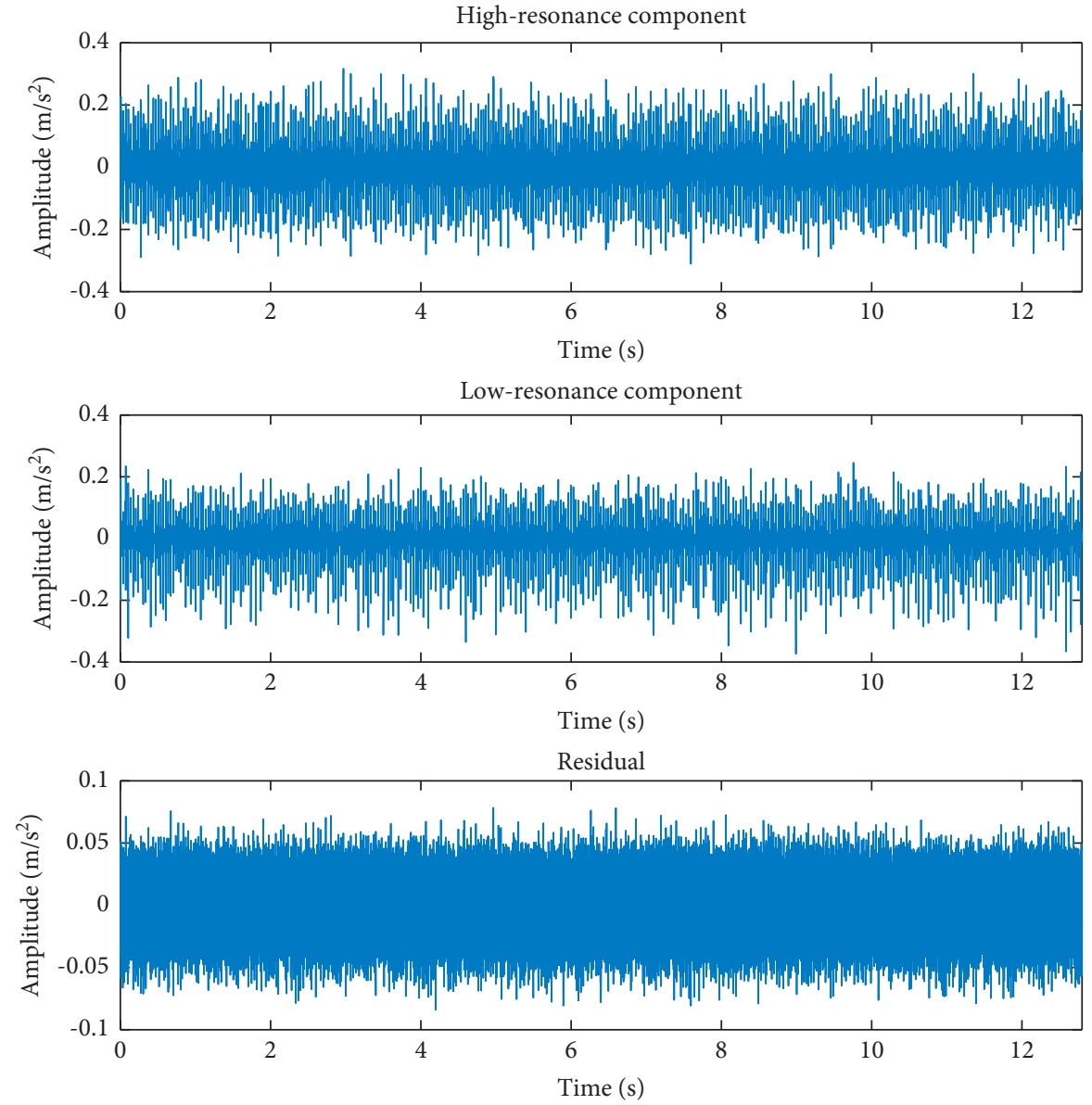

(b)

FIgURe 13: Continued. 

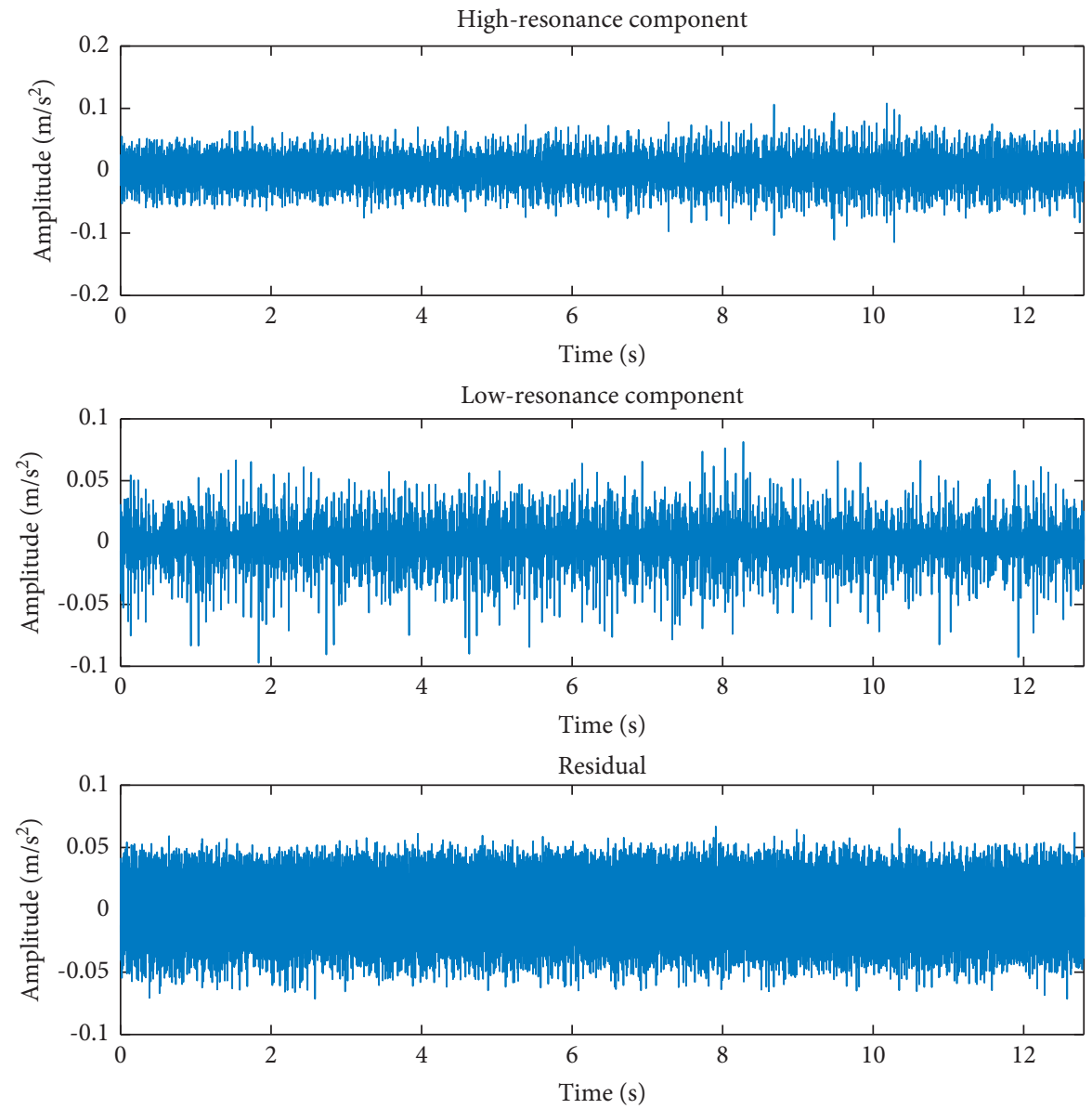

(c)

FIgURE 13: Resonance-based sparse signal decomposition of fault signals. (a) Resonance-based sparse signal decomposition result of vibration signal of abrasion. (b) Resonance-based sparse signal decomposition result of vibration signal of notched gear. (c) Resonancebased sparse signal decomposition result of vibration signal of tooth fracture.

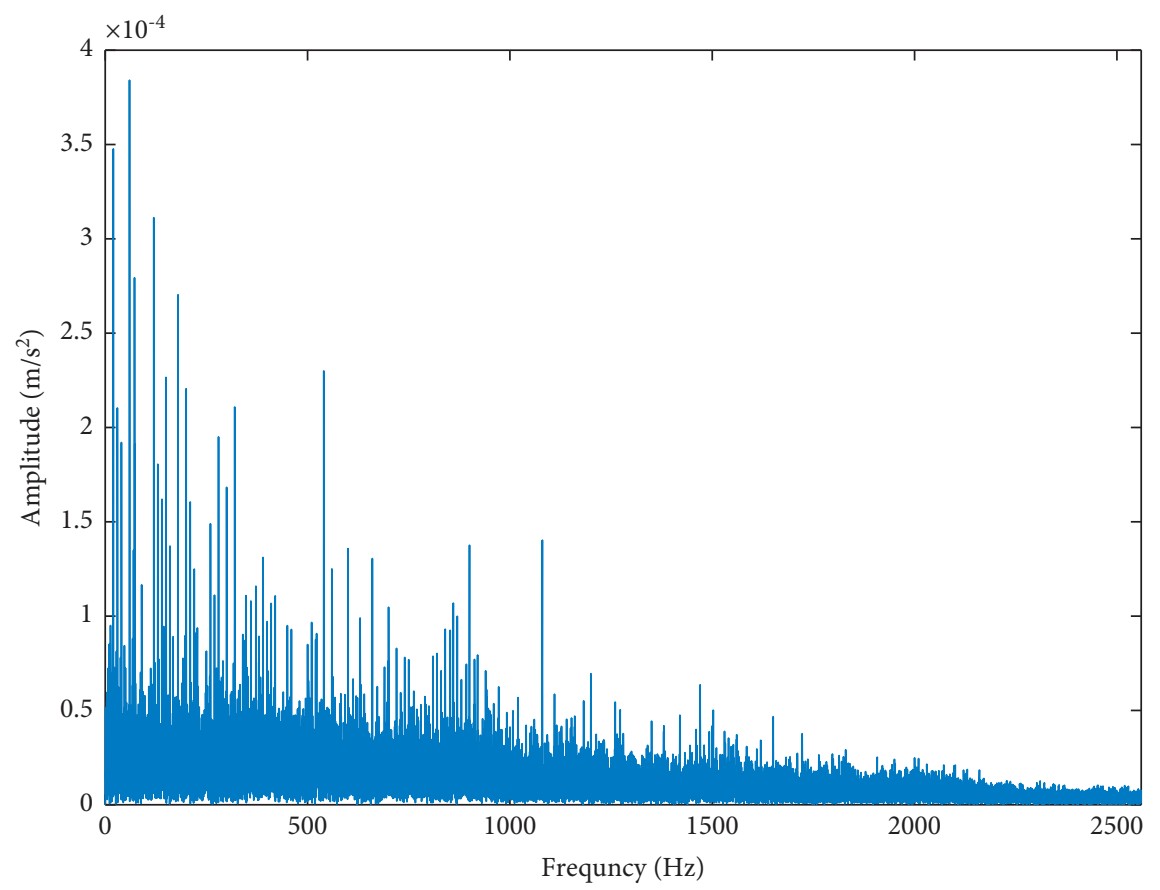

(a)

FIgURE 14: Continued. 


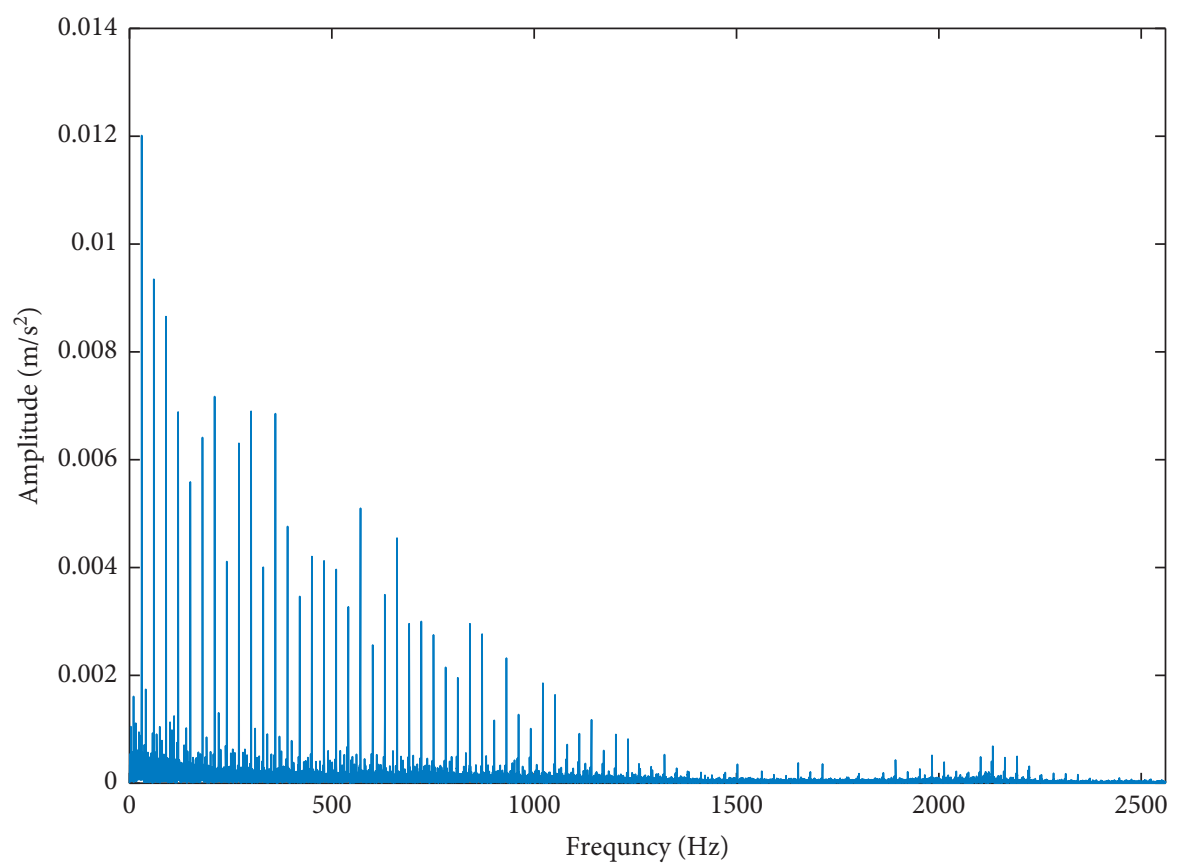

(b)

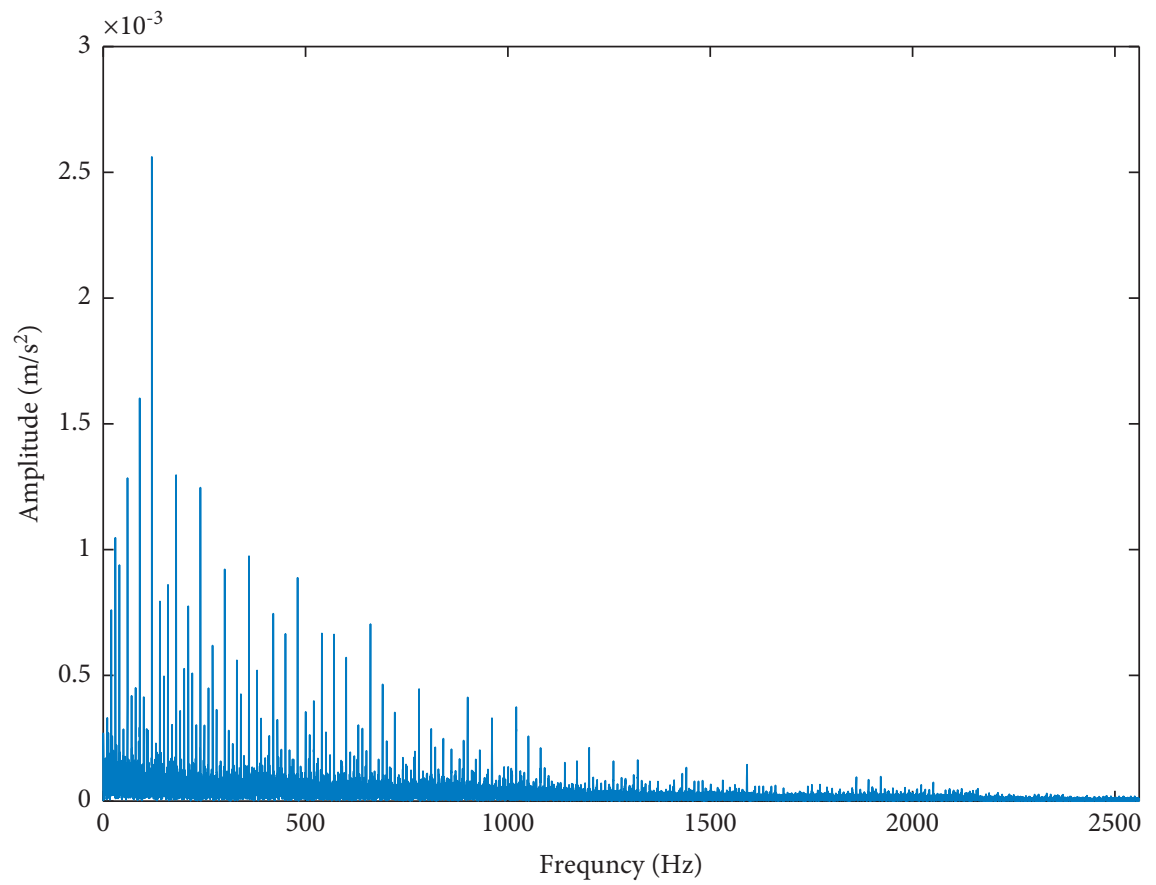

(c)

Figure 14: Envelope spectrum of low-resonance component. (a) Envelope spectrum of low-resonance component for abrasion. (b) Envelope spectrum of low-resonance component for notched gear. (c) Envelope spectrum of low-resonance component for tooth fracture. 


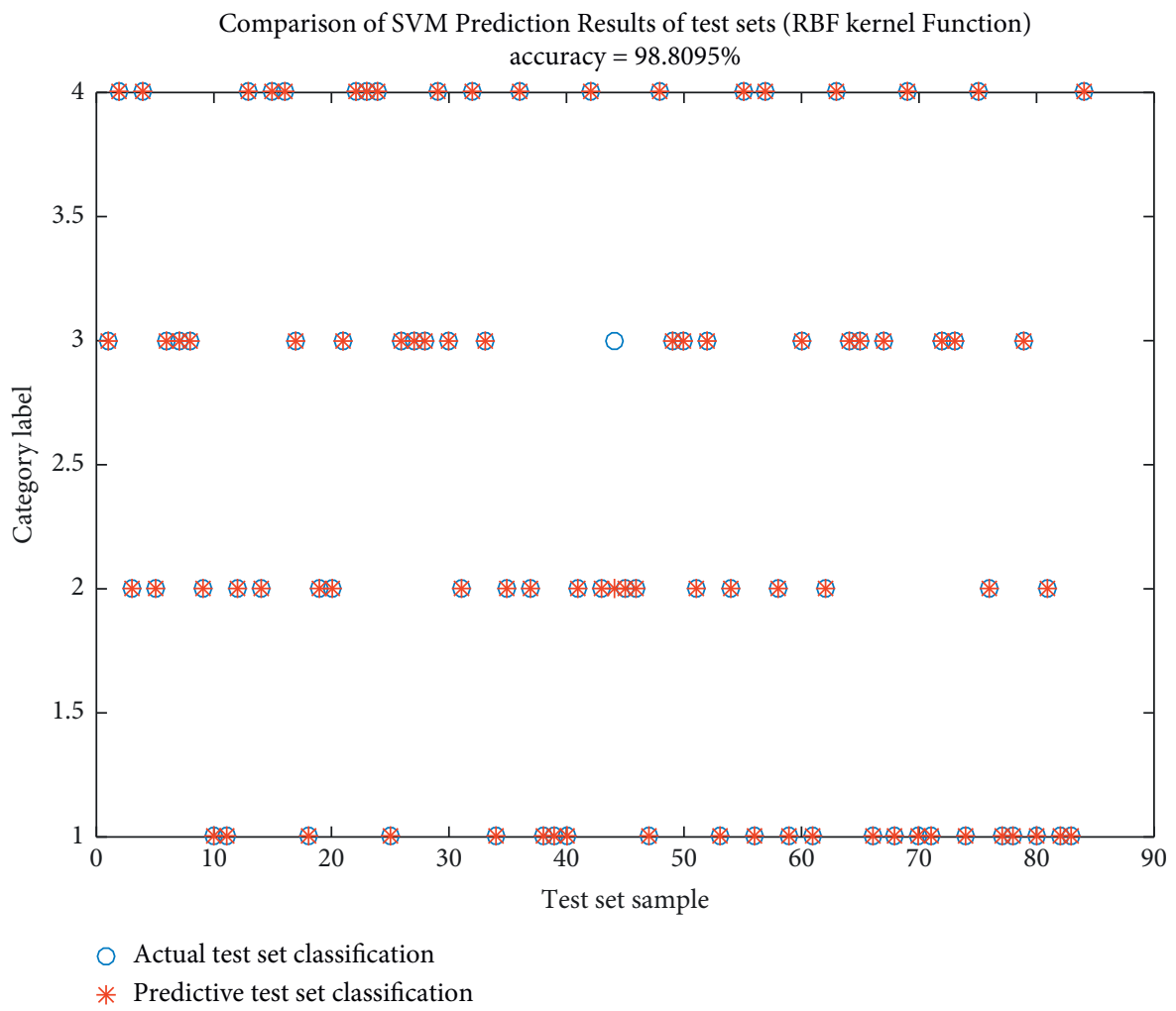

(a)

Actual classification and predictive classification of test sets Accuracy $=85.7143 \%$

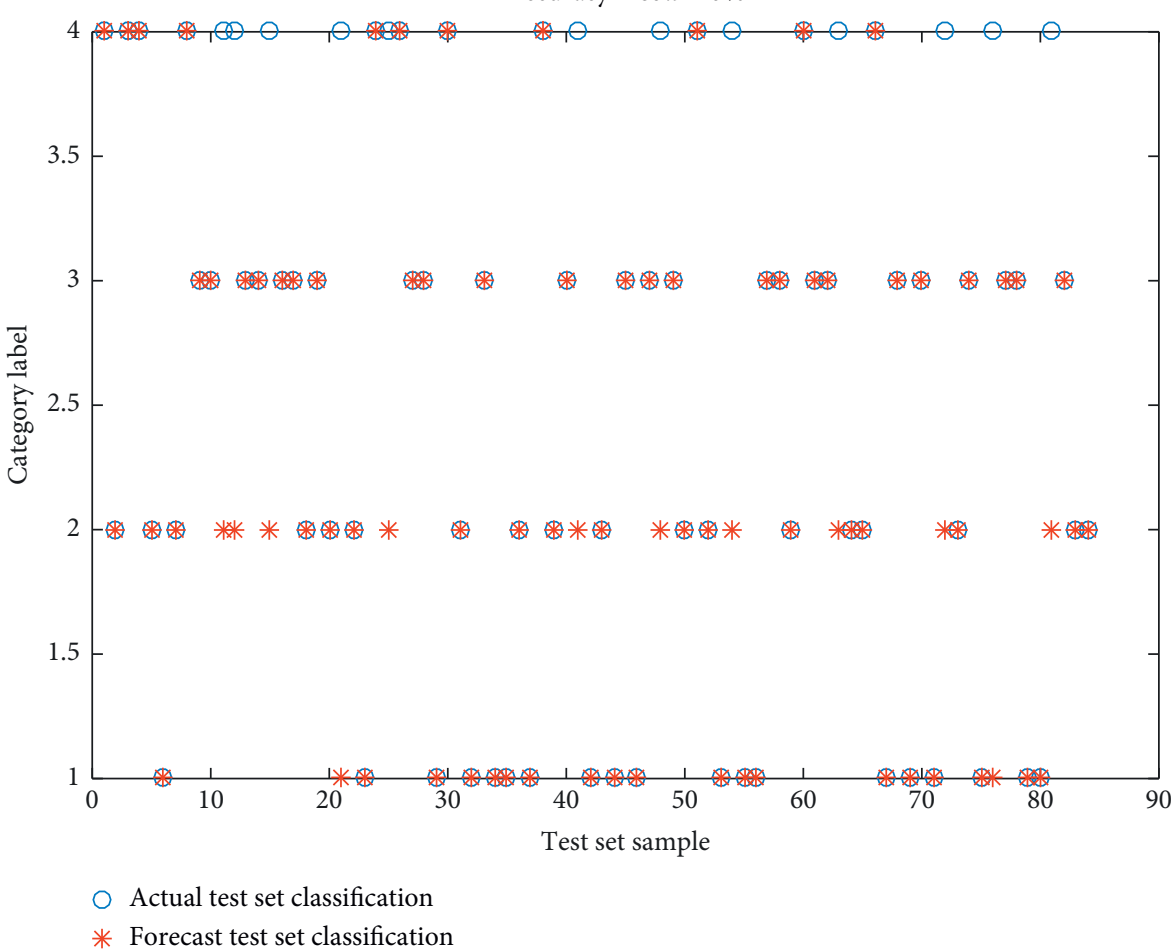

(b)

FIGURE 15: Identification result of CV-SVM model. (a) Identification result of CV-SVM model of processed signal. (b) Identification result of CV-SVM of model of original signals. 
recognition are shown in Figure 15, and the final accuracy rate of the proposed method is $98.8095 \%$, while the accuracy rate of CV-SVM which uses the original vibration data for feature extraction is only $85.7143 \%$. As shown in Figure 15, compared with the optimization method, the effectiveness of the original method is not as ideal as that of the proposed method.

\section{Conclusion}

In this paper, an improved gearbox fault diagnosis method based on optimized resonant sparse signal decomposition combined with CV-SVM is developed. Through simulation and experimental verification, the following conclusions are drawn:

(1) The atom search optimization algorithm is employed in the optimization of the resonance-based sparse signal decomposition. The kurtosis of the low-resonance component is taken as the optimization objective parameter, with which the decomposition ability of the resonant sparse signal decomposition method for fault signals was improved. The effectiveness of this method is verified with simulation and experiments.

(2) The power spectrum entropy, singular spectrum entropy, and time-domain energy entropy are used as eigenvalues to apply to CV-SVM, which distinctively improved the accuracy of gearbox fault pattern recognition.

(3) The integrated model of ASO-based resonance sparse signal decomposition and CV- SVM is effective and feasible for gearbox fault diagnosis.

In the future, we will further explore the case of gearbox under compound fault mode, and the effectiveness of the proposed method could be further evaluated or optimized.

\section{Nomenclature}

RSSD: Resonance-based sparse signal decomposition

SVM: Support vector machine

ASO: Atom search optimization

TSA: Time synchronized average

EMD: Empirical mode decomposition

EEMD: Ensemble empirical mode decomposition

LMD: Local mean decomposition

VMD: Variational mode decomposition

ITD: Intrinsic time-scale decomposition

SK: $\quad$ Spectral kurtosis

CWT: Continuous wavelet transformation

ASR: Adaptive stochastic resonance

DTW: Dynamic time warping

TQWT: Tunable Q-factor wavelet transform

SALSA: Separation augmented Lagrange contraction algorithm

$x$ : $\quad$ Gearbox vibration signal

$x_{1}: \quad$ The high-resonance component $x_{2}: \quad$ The low-resonance component

$H_{0}(\omega)$ : The low-resonance filter

$H_{1}(\omega)$ : The high-resonance filter

$\alpha: \quad$ The low-pass scale factor

$\beta$ : $\quad$ The high-pass scale factor

Q: $\quad$ Quality factor

$Q_{1}$ : High-quality factor

$Q_{2}$ : Low-quality factor

$R: \quad$ Redundancy factor

$r_{1}$ : The redundancy of high-resonance component

$r_{2}$ : The redundancy of low-resonance component

$L_{\max }$ : The maximum number of decomposition layers

$S_{1}, S_{2}$ : The overcomplete basis function libraries

$\lambda_{1}, \lambda_{2}$ : The regularization parameters

$\mu$ : $\quad$ The mean value of the vibration signal

$\sigma: \quad$ The standard deviation of the vibration signal

E: $\quad$ The mathematical expectation

$\delta: \quad$ The depth weighting

$\gamma: \quad$ The multiplier weight

$T_{\max }: \quad$ The maximum number of iterations

$h_{i j}(t)$ : The distance between two atoms

$m_{i}(t)$ : The mass of the $i-$ th atom after the $t-$ th iteration

$x_{i}^{d}(t)$ : The position of the $i-$ th atom after the $t-$ th iteration

$v_{i}^{d}(t): \quad$ The velocity of the $i-$ th atom after the $t-$ th iteration

$a_{i}^{d}(t)$ : The acceleration of the $i-$ th atom after the $t-$ th iteration

$\zeta_{i}: \quad$ Relaxation variable

sgn: The function

$w$ : Weight vector

$b: \quad$ Bias

$c_{i}^{*}: \quad$ The optimal solution of $c_{i}$

$b^{*}: \quad$ Threshold value of classification

$\chi$ : $\quad$ Samples to be classified

$M(x)$ : Classification function

$c$ : Penalty coefficient

$g$ : Kernel function.

\section{Data Availability}

The data used to support the findings of this study are available from the corresponding author upon request.

\section{Conflicts of Interest}

The authors declare that they have no conflicts of interest.

\section{Acknowledgments}

This research was supported by project of National Natural Science Foundation of China (no. 52075050), Natural Science Research Project of Universities in Jiangsu Province (no. 19KJA430004), and Jiangsu Key Laboratory of Green Process Equipment. 


\section{References}

[1] J. Guo, D. Zhen, H. Li, Z. Shi, F. Gu, and A. D. Ball, "Fault detection for planetary gearbox based on an enhanced average filter and modulation signal bispectrum analysis," ISA Transactions, vol. 101, pp. 408-420, 2020.

[2] T. Wang, Q. Han, F. Chu, and Z. Feng, "Vibration based condition monitoring and fault diagnosis of wind turbine planetary gearbox: a review," Mechanical Systems and Signal Processing, vol. 126, pp. 662-685, 2019.

[3] B. Assaad, M. Eltabach, and J. Antoni, "Vibration based condition monitoring of a multistage epicyclic gearbox in lifting cranes," Mechanical Systems and Signal Processing, vol. 42, no. 1-2, pp. 351-367, 2014.

[4] G. Dalpiaz, A. Rivola, and R. Rubini, "Effectiveness and sensitivity of vibration processing techniques for local fault detection in gears," Mechanical Systems and Signal Processing, vol. 14, no. 3, pp. 387-412, 2000.

[5] Z. Feng, X. Lin, and M. J. Zuo, "Joint amplitude and frequency demodulation analysis based on intrinsic time-scale decomposition for planetary gearbox fault diagnosis," Mechanical Systems and Signal Processing, vol. 72-73, pp. 223-240, 2016.

[6] J. Antoni, "The spectral kurtosis: a useful tool for characterising non-stationary signals," Mechanical Systems and Signal Processing, vol. 20, no. 2, pp. 282-307, 2006.

[7] B. P. Tang, W. Y. Liu, and T. Song, "Wind turbine fault diagnosis based on Morlet wavelet transformation and WignerVille distribution," Journal of Vibration and Acoustics, vol. 35, no. 12, 2010.

[8] Y. Lei, D. Han, J. Lin, and Z. He, "Planetary gearbox fault diagnosis using an adaptive stochastic resonance method," Mechanical Systems and Signal Processing, vol. 38, no. 1, pp. 113-124, 2013.

[9] Z. Feng, M. Liang, Y. Zhang, and S. Hou, "Fault diagnosis for wind turbine planetary gearboxes via demodulation analysis based on ensemble empirical mode decomposition and energy separation," Renewable Energy, vol. 47, pp. 112-126, 2012.

[10] I. W. Selesnick, "Resonance-based signal decomposition: a new sparsity-enabled signal analysis method," Signal Processing, vol. 91, no. 12, pp. 2793-2809, 2011.

[11] D. Juan, L. Yan, T. Xiao, Z. Yu, and C. G. Chu, "Periodic impulse signal separation based on resonance-based sparse signal decomposition and its application to the fault detection of rolling bearing," Measurement and Control, vol. 53, no. 3-4, pp. 601-612, 2020.

[12] Y. S. Sun, D. J. Yu, X. M. Chen, and R. Li, "Order domain analysis based on resonance-based sparse signal decomposition and its application to gear fault diagnosis," Journal of Vibration and Shock, vol. 32, no. 16, pp. 88-94, 2013.

[13] B. P. Tang, X. Y. Xiong, M. H. Zhao, and Q. Tan, "A multiresonance component fusion based convolutional neural network for fault diagnosis of planetary gearboxes," Journal of Vibration, Measurement \& Diagnosis, vol. 40, no. 3, pp. 507-512, 2020.

[14] X. Li, J. Cheng, H. Shao, K. Liu, and B. Cai, "A fusion CWSMM-based framework for rotating machinery fault diagnosis under strong interference and imbalanced case," IEEE Transactions on Industrial Informatics, p. 1, 2021.

[15] J. Xia and M. P. Jia, "Rolling bearing fault diagnosis with a resonance-based sparse decomposition and squirrel optimization algorithm," Journal of Vibration and Shock, vol. 40, no. 4, pp. 250-254, 2021.

[16] Y. Huang, S. Tong, Z. Tong, and F. Cong, "Signal identification of gear vibration in engine-gearbox systems based on auto- regression and optimized resonance-based signal sparse decomposition," Sensors, vol. 21, no. 5, p. 1868, 2021.

[17] X. Wang, P. Xie, Y. G. Guo, X. Wu, G. Q. Jiang, and Q. He, "Impulse fault signature extraction based on multi-dictionary resonance-based sparse signal decomposition," China Mechanical Engineering, vol. 30, no. 20, pp. 2456-2462, 2019.

[18] W. Huang, H. Sun, and W. Wang, "Resonance-based sparse signal decomposition and its application in mechanical fault diagnosis: a review," Sensors, vol. 17, no. 6, p. 1279, 2017.

[19] F. J. Yu, F. X. Zhou, and B. K. Yan, "Bearing initial fault feature extraction via sparse representation based on dictionary learning," Journal of Vibration and Shock, vol. 35, no. 6, pp. pp181-186, 2016.

[20] W. Zhao, L. Wang, and Z. Zhang, "A novel atom search optimization for dispersion coefficient estimation in groundwater," Future Generation Computer Systems, vol. 91, pp. 601-610, 2019.

[21] L. J. Li, Z. S. Zhang, and Z. J. He, "Research of mechanical system fault diagnosis based on support vector data description," Journal of Xi'an Jiaotong University, vol. 37, no. 9, pp. 910-913, 2003.

[22] X. F. Wang, J. Qiu, and G. J. Liu, "Discrete particle swarm optimization algorithm for gearbox fault symptom selection," Journal of Aerospace Power, vol. 20, no. 6, pp. 969-972, 2005.

[23] E. Duarte and J. Wainer, "Empirical comparison of crossvalidation and internal metrics for tuning SVM hyperparameters," Pattern Recognition Letters, vol. 88, pp. 6-11, 2017.

[24] G. Cheng, X. Chen, H. Li, P. Li, and H. Liu, "Study on planetary gear fault diagnosis based on entropy feature fusion of ensemble empirical mode decomposition," Measurement, vol. 91, pp. 140-154, 2016.

[25] B. Wang, M. Diao, and K. Song, "Fault diagnosis of hydrogen sensor based on wavelet singular entropy and relevance vector machine," Electric Machines and Control, vol. 19, no. 1, pp. 96-101, 2015.

[26] Y. Feng, X. D. Huang, R. J. Hong, and J. Chen, "Incipient fault diagnosis of large-size slewing bearings based on circular domain analysis," Journal of Vibration and Shock, vol. 36, no. 9, pp. 108-105, 2017. 